\title{
Classificação de Gliomas Utilizando Índices de Biodiversidade e de Diversidade Filogenética em Imagens por Ressonância Magnética Através de uma Abordagem Radiomics
}

\author{
Fernanda Duarte $^{1}$, Aristófanes C. Silva ${ }^{2}$, Marcelo Gattass ${ }^{1}$, Antonino C. dos S. Neto ${ }^{2}$ \\ ${ }^{1}$ Departamento de Informática - Pontifícia Universidade Católica do Rio de Janeiro \\ (DI/PUC-Rio) \\ Rio de Janeiro, Brasil \\ ${ }^{2}$ Departamento de Engenharia Elétrica - Universidade Federal do Maranhão \\ (DEE/UFMA) \\ Maranhão, Brasil
}

\begin{abstract}
Gliomas are among the most common malignant brain tumors. They can be classified into low-grade and high-grade gliomas and their early identification is crucial for treatment direction. Using a radiomics approach, the present work proposes the use of biodiversity and phylogenetic diversity biology indices to handle the glioma classification problem. The proposed method presented promising results, with AUC, accuracy, sensitivity and specificity of 0,926, 0,902, 0,962 and 0,733, respectively.
\end{abstract}

Resumo. Gliomas estão entre os tumores cerebrais malignos mais comuns. Eles podem ser classificados entre gliomas de baixo e alto grau e sua identificação precoce é fundamental para o direcionamento do tratamento aplicado. Utilizando uma abordagem radiomics, o presente trabalho propõe o uso de índices de biodiversidade e de diversidade filogenética, definidos no campo da biologia, no problema de classificação de gliomas. O método proposto apresentou resultados promissores, com AUC, acurácia, sensibilidade e especificidade de 0,926, 0,902, 0,962 e 0,733, respectivamente.

\section{Introdução}

Gliomas são tumores do sistema nervoso central que se originam nas células gliais e correspondem a aproximadamente $74.6 \%$ dos tumores cerebrais malignos [NBTS 2017]. Eles são comumente classificados entre gliomas de baixo grau (LGG, low grade gliomas) e de alto grau (HGG, high grade gliomas). Gliomas de baixo grau compreendem os graus I e II definidos pela Organização Mundial de Saúde (WHO, World Health Organization), enquanto que os de alto grau incluem os graus III e IV [Louis et al. 2007, Louis et al. 2016].

Gliomas de grau II, apesar de apresentarem um melhor prognóstico em relação aos estágios superiores, podem se desenvolver rapidamente para os graus III e IV, que por sua vez apresentam uma taxa de sobrevivência extremamente baixa [Claus et al. 2015]. Sendo assim, a classificação entre HGG e LGG feita por médicos especialistas é de extrema importância para a formulação do prognóstico e direcionamento do tratamento dos pacientes. 
Atualmente, os principais meios utilizados para a realização do diagnóstico de gliomas envolvem a realização de exames de imagem, como imagens por ressonância magnética (MRI, magnetic resonance imaging), tomografia computadorizada (CT, computed tomography) e tomografia por emissão de pósitrons (PET, positron emission tomography), e exames de biópsia, que consistem na retirada de uma porção do tumor para posterior análise em microscópio.

Com o avanço cada vez mais notável de algoritmos de aprendizagem de máquina e a crescente disponibilidade de dados médicos, muito estudos que combinam métodos computacionais e conhecimentos da medicina têm surgido em uma tentativa de criar técnicas que auxiliem no diagnóstico e tratamento de doenças. Um dos tópicos que mais tem ganhado destaque nesse contexto está relacionado à análise e interpretação de imagens médicas e deu origem a um procedimento chamado radiomics, cuja principal premissa é a utilização de atributos quantitativos extraídos de imagens médicas digitalizadas como eficientes indicadores de características genéticas e fisiopatológicas capazes de auxiliar nas decisões tomadas por especialistas [Gillies et al. 2016]. Além de oferecer informações que muitas vezes não são percebidas pelo olho humano, tais atributos extraídos por métodos computacionais podem oferecer uma excelente alternativa aos meios invasivos atualmente utilizados para a confirmação de determinados diagnósticos. Mais ainda, o uso do radiomics pode otimizar o tempo que especialistas gastam para analisar o grande volume de exames que recebem.

Muitos estudos recentes utilizaram atributos radiomics baseados em imagens por ressonância magnética para lidar com o problema de classificação de gliomas, fundamentando-se na ideia de que gliomas de graus mais elevados tendem a apresentar uma estrutura mais heterogênea [McGranahan and Swanton 2015] e utilizando a extração de características de textura e forma para mensurar tal heterogeneidade. Um estudo conduzido por [Zhang et al. 2017] comparou a eficiência de diversos modelos de machine learning na classificação de gliomas utilizando dados de ressonância magnética. Outro estudo [Chen et al. 2018] propôs-se a desenvolver um sistema automático para classificação de gliomas, aplicando técnicas de deep learning para realizar uma segmentação automática das lesões anterior à extração de atributos radiomics.

Um artigo recentemente publicado [Cho et al. 2018] apresentou um modelo de classificação entre gliomas de baixo e alto grau utilizando 5 atributos radiomics extraídos da base de dados de MRI fornecida pelo desafio BraTS 2017, mesma base oferecida pelo desafio em 2018 e utilizada em nosso estudo. Os valores de acurácia e AUC obtidos foram de 0,8877 e 0,9213 , respectivamente. O presente trabalho se propõe a trazer conceitos do campo da biologia para a área de imagens médicas, introduzindo atributos radiomics ainda não utilizados no problema de classificação de gliomas entre LGG e HGG. Através das imagens por ressonância magnética fornecidas pelo desafio BraTS 2018 (Multimodal Brain Tumor Segmentation Challenge 2018) [Menze, B. H. et al. 2015, Bakas et al. 2017] são calculados índices de biodiversidade e de diversidade filogenética, que serão utilizados como atributos em algoritmos de machine learning de classificação. Trabalhos anteriores [de Carvalho Filho et al. 2017, Neto et al. 2018] utilizaram índices filogenéticos em problemas relacionados a nódulos pulmonares, apresentando excelentes resultados. Do mesmo modo, os modelos aqui apresentados mostram que tais índices, em conjunto com índices de biodiversidade, possuem um grande poder discriminativo no 
problema de classificação de gliomas, gerando resultados comparáveis àqueles já existentes.

\section{Base de Dados}

Foram utilizados os dados de treinamento oferecidos pelo desafio BraTS 2018 [Menze, B. H. et al. 2015, Bakas et al. 2017]. Os dados foram fornecidos por 19 instituições e correspondem a exames de MRI de 285 indivíduos, com presença confirmada de gliomas, sendo 210 indivíduos com HGG e 75 com LGG. Cada exame apresenta quatro sequências distintas de ressonância: ponderada em T1 (T1), ponderada em T1 pós-contraste (T1ce), ponderada em T2 (T2) e inversão-recuperação atenuante de fluido ponderada em T2 (FLAIR, Fluid Attenuated Inversion Recovery). Cada modalidade de MRI corresponde a uma imagem volumétrica de dimensões 240x240x155 e a Figura 1 ilustra alguns exemplos.
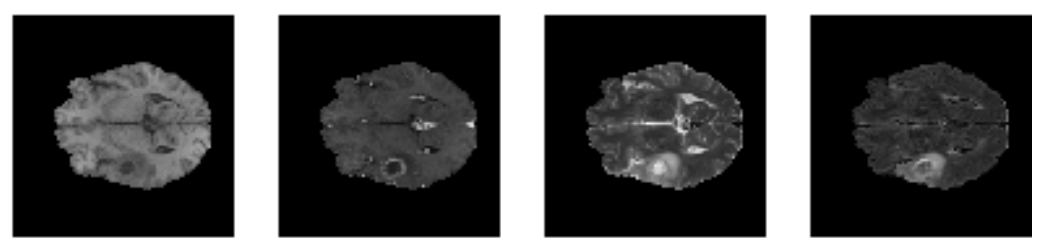

Figura 1. Exemplos de fatias extraídas das diferentes modalidades de MRI. Da esquerda para a direita: T1, T1ce, T2 e FLAIR

As lesões de cada indivíduo foram anotadas manualmente e disponibilizadas em um arquivo à parte, fornecendo as posições dos voxels correspondentes ao volume de interesse a ser extraído de cada exame. Antes de serem distribuídas, todas as imagens foram submetidas a etapas de pré processamento que consistiram em um co-registro para um modelo anatômico de referência, uma interpolação para a mesma resolução $\left(1 \mathrm{~mm}^{3}\right)$ e na extração da caixa craniana.

\section{Metodologia Proposta}

A Figura 2 ilustra a metodologia adotada pelo presente estudo. A partir da base de dados obtida, realizou-se um pré-processamento (Seção 3.1) seguido pela etapa de obtenção de atributos (Seção 3.2), em que foram definidas regiões de interesse e adaptações necessárias para o cálculo dos índices de biodiversidade (Seção 3.2.1) e de diversidade filogenética (Seção 3.2.2). Em seguida foi aplicado um algoritmo de seleção de atributos (Seção 3.3), com o objetivo de melhorar a eficácia do modelo final. Por fim, foi realizada a etapa de classificação (Seção 3.4), na qual foi avaliado o desempenho de três algoritmos distintos de aprendizagem de máquina utilizando uma validação cruzada 5-fold.

\subsection{Pré-processamento}

Os dados utilizados são provenientes de diferentes instituições e, consequentemente, são exames produzidos por diferentes aparelhos. Sendo assim, para cada modalidade de ressonância, foi aplicado um algoritmo de histogram matching [Gonzalez and Woods 2006] sobre todas as imagens, tomando a imagem de um indivíduo como referência, de modo a garantir que todas apresentem intervalos de cinza semelhantes. A imagem de referência foi escolhida utilizando como critério a quantidade de fatias pretas (ou seja, com todos 


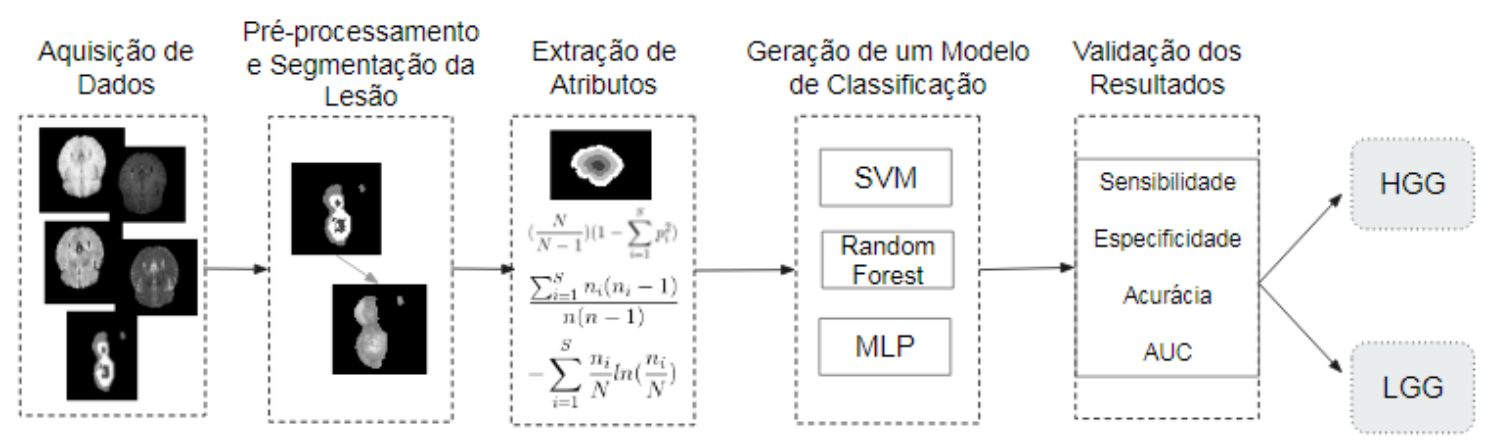

Figura 2. Metodologia proposta.

os pixels assumindo o valor 0) presentes em cada imagem volumétrica, escolhendo o indivíduo cujos exames apresentaram a menor quantidade. Em seguida, utilizando as anotações de segmentação fornecidas pela base de dados, as lesões foram extraídas de cada imagem volumétrica para serem utilizadas nas próximas etapas de extração e seleção de atributos.

\subsection{Extração de atributos}

Para a extração dos atributos, foram definidas 4 regiões intratumorais, cada uma correspondendo a aproximadamente $1 / 4$ do volume da lesão, com o objetivo de capturar características específicas de partes mais externas ou internas do tumor. A Figura 3 ilustra uma fatia da imagem de anotação após a divisão em camadas, na qual cada camada é representada por um tom de cinza diferente. Através dos índices definidos nas seções a seguir, foram calculados atributos de cada região separadamente, sendo obtidos ao todo 272 atributos (12 índices de biodiversidade e 5 de diversidade filogenética calculados sobre 4 regiões intratumorais para 4 modalidades de MRI ) para serem utilizados como dados de entrada dos algoritmos de classificação.

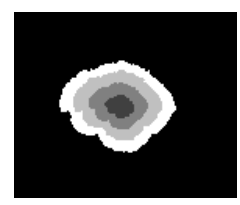

Figura 3. Exemplo de fatia de uma imagem de anotação após a divisão por camadas.

\subsection{1. Índices de Biodiversidade}

Índices de biodiversidade são muito utilizados para se medir a diversidade de espécies presentes em determinada região. De acordo com [Morris et al. 2014], a biodiversidade representa a variedade e heterogeneidade de organismos em qualquer nível de organização de seres vivos, desde moléculas até ecossistemas. Levando em conta a noção de que heterogeneidade intratumoral geralmente está associada a um pior prognóstico [Gillies et al. 2016] e que, portanto, gliomas de mais alto grau tendem a apresentar uma estrutura mais heterogênea, fez-se uma adaptação de índices da biologia para o contexto de imagens [de Carvalho Filho et al. 2017, Neto et al. 2018] de modo a tentar utilizar a 
ideia de biodiversidade para capturar e mensurar as diferentes estruturas características de cada tumor.

Utilizando diferentes índices de biodiversidade, o objetivo é mostrar que lesões com estruturas mais heterogêneas apresentam uma maior medida de diversidade em relação a estruturas mais homogêneas. A adaptação dos índices para o contexto de imagens médicas foi baseada no trabalho publicado por [Neto et al. 2018], em que cada voxel corresponde a um indivíduo e o valor assumido por ele, à sua espécie. Sendo assim, o conjunto de voxels que compõem a lesão define a comunidade estudada. O raciocínio utilizado está esquematizado na Tabela 1 a seguir.

Tabela 1. Correspondência entre os diferentes contextos

\begin{tabular}{|c||c|}
\hline Biologia & Metodologia proposta \\
\hline Comunidade & Volume de interesse nos dados de MRI \\
\hline Indivíduo & Voxel \\
\hline Espécie & Valor do voxel \\
\hline
\end{tabular}

A seguir são descritos os 12 índices de biodiversidade [Karydis and Tsirtsis 1996, Campos and Isaza 2009, Lamb et al. 2009] utilizados como atributos em nosso estudo.

1. Quantidade de espécies $(S)$ : Corresponde à quantidade de diferentes espécies presentes na comunidade estudada.

2. Quantidade de indivíduos $(N)$ : Número total de indivíduos na comunidade.

3. Índice de Margalef $\left(D_{M g}\right)$ : É uma medida de riqueza de espécies baseada na hipótese de que existe uma relação linear entre a quantidade total de espécies e o logaritmo da quantidade de indivíduos. O índice é dado por

$$
D_{M g}=\frac{S-1}{\ln N}
$$

4. Índice de Menhinck $\left(D_{M n}\right)$ : Também é uma medida de riqueza de espécies, mas apresenta menor variação entre amostras de diferentes comunidades, se comparado ao índice anterior.

$$
D_{M n}=\frac{S}{\sqrt{N}}
$$

5. Índice de Odum $\left(D_{O}\right)$ : Corresponde à quantidade de espécies por mil indivíduos multiplicada pela quantidade total de indivíduos.

$$
D_{O}=\frac{S N}{1000}
$$

6. Índice de Hulbert $(P I E)$ : É a probabilidade de que dois indivíduos escolhidos ao acaso, em uma amostra aleatória de determinada comunidade, pertençam a espécies diferentes.

$$
P I E=\left(\frac{N}{N-1}\right)\left(1-\sum_{i=1}^{S} p_{i}^{2}\right)
$$

em que $p_{i}$ é a fração de indivíduos dentro da amostra pertencentes à espécie $i$, ou a probabilidade de escolher um indivíduo ao acaso e ele pertencer à espécie $i$. 
7. Índice de McNaughton $(I)$ : Corresponde à porcentagem das duas espécies com maior quantidade de indivíduos na amostra da comunidade estudada.

$$
I=\frac{n_{1}+n_{2}}{N} 100
$$

Sendo $n_{1}$ e $n_{2}$ a quantidade de indivíduos pertencentes à primeira e à segunda espécies mais populosas, respectivamente.

8. Índice de Simpson $\left(D_{S}\right)$ : Indica a probabilidade de que dois indivíduos escolhidos aleatoriamente em uma amostra pertençam à mesma espécie.

$$
D_{S}=\frac{\sum_{i=1} n_{i}\left(n_{i}-1\right)}{N(N-1)}
$$

Em que $n_{i}$ é a quantidade de indivíduos pertencentes à espécie $i$.

9. Índice de Shannon $\left(H^{\prime}\right)$ : Mede o grau de incerteza médio ao se tentar prever a qual espécie pertence determinado indivíduo escolhido aleatoriamente em uma amostra.

$$
H^{\prime}=-\sum_{i=1}^{S} \frac{n_{i}}{N} \ln \left(\frac{n_{i}}{N}\right)
$$

10. Índice de Uniformidade (Evenness index, E) : Mede o quão uniforme é a distribuição de indivíduos entre as espécies existentes na amostra. Seu valor máximo é atingido quando todas as espécies possuem a mesma quantidade de indivíduos.

$$
E=\frac{H^{\prime}}{H_{\text {max }}^{\prime}}
$$

Em que $H_{\text {max }}^{\prime}$ corresponde ao valor máximo do índice de Shannon.

11. Índice de Redundância (Redundancy Index, $R$ ) : A redundância é inversamente proporcional à quantidade de espécies, podendo ser pensada como uma medida do quanto a abundância da amostra pode ser explicada por uma ou mais espécies.

$$
R=\frac{H_{\max }^{\prime}-H^{\prime}}{H_{\max }^{\prime}-H^{\prime} \min }
$$

Sendo $H_{\text {min }}^{\prime}$ o valor mínimo do índice de Shannon.

12. Índice geométrico $(B)$ : O índice compara um sistema de $S$ espécies com um sistema de $S+k$ espécies, usando para tal comparação a relação entre o volume de duas esferas de raio $r$ existentes em espaços de dimensão $S$ e $S+k$, respectivamente.

$$
\begin{gathered}
B_{k}(S, r)=\frac{V_{s}(r)}{V_{s+k}(r)}=\alpha_{k}(S) \beta_{k}(r), \quad r \neq 0 \\
V_{s}(r)=\frac{\pi^{S / 2}}{\Gamma((S / 2)+1)} r^{S}
\end{gathered}
$$

Em que $V_{s}(r)$ é o volume de uma esfera com raio $r$ em um espaço $S$-dimensional e $\Gamma(x)$ é a função Gamma com as seguintes propriedade: $\Gamma(u+1)=u \Gamma(u)$, $\Gamma(1)=1, \Gamma(1 / 2)=\sqrt{\pi}, \Gamma(3 / 2)=(1 / 2) \sqrt{\pi}, \Gamma(5 / 2)=(3 / 4) \sqrt{\pi}$ etc. $\alpha_{k}(S)$ e $\beta_{k}(r)$ são definidos por

$$
\alpha_{k}(S):=\frac{\Gamma((S+k+2) / 2)}{\pi^{k / 2} \Gamma((S+2) / 2)}, \quad \beta_{k}(r):=\frac{1}{r^{k}}, \quad r=\sqrt{\sum_{i=1}^{S} p_{i}^{2}}
$$




\subsection{2. Índices de Diversidade Filogenética}

Ao analisar a diversidade de espécies em determinada comunidade, é importante notar que, além de existirem diferentes espécies, existe também uma noção de distância evolutiva entre elas, cujo principal objetivo é mensurar o quão diferentes duas espécies são entre si. Uma das formas de se calcular essa distância evolutiva é através das relações filogenéticas que descrevem a evolução das espécies ao longo do tempo, tentando estabelecer conexões entre cada espécie e seus ancestrais. A representação dessas relações pode ser feita através das chamadas árvores filogenéticas, em que as ramificações representam a forma como espécies, ou grupo de espécies, evoluíram a partir de seus ancestrais comuns. Assim, pode-se dizer que duas espécies possuem uma curta distância filogenética se elas possuem um ancestral comum mais recente, ou uma longa distância caso contrário.

Inspirado por resultados publicados por estudos recentes [de Carvalho Filho et al. 2017, Neto et al. 2018] que utilizam índices filogenéticos em problemas envolvendo a classificação de nódulos pulmonares, nosso estudo combina índices de diversidade filogenética aos índices de biodiversidade anteriormente descritos para serem usados como atributos no problema de classificação de gliomas entre HGG e LGG. Para capturar a noção de distância entre espécies em nosso contexto de imagens médicas, foram reproduzidos os mesmos procedimentos utilizados nos trabalhos citados anteriormente. Tais procedimentos são explicados a seguir. Utilizando as adaptações da Tabela 1 e um cladograma, como o indicado na Figura 4, representa-se através de nós terminais (ou folhas) cada espécie existente na região de interesse analisada e utiliza-se arestas para indicar as distâncias entre elas. Os nós internos representam os ancestrais comuns entre as espécies. Deste modo, a distância $d_{i j}$ entre duas espécies $i$ e $j$ é dada pela quantidade de arestas existentes no menor caminho que vai do nó $i$ ao nó $j$.

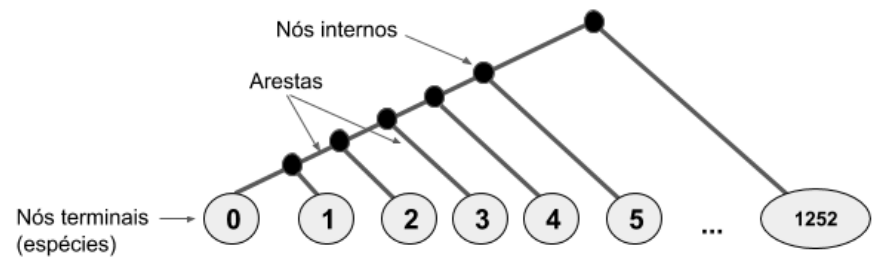

Figura 4. Exemplo de relações filogenéticas representadas por um cladograma. Neste exemplo, o valor máximo de voxel encontrado no volume de interesse é 1252. trabalho:

A seguir são descritos os 5 índices de diversidade filogenética utilizados em nosso

1. Entropia quadrática intensiva $(J)$ : Fornece a distância taxonômica média entre duas espécies selecionadas ao acaso [Izsák and Papp 2000].

$$
J=\frac{\sum_{i=0}^{N} \sum_{j=0}^{N} d_{i j}}{S^{2}}
$$

2. Entropia quadrática extensiva $(F)$ : É dada pela soma total das distâncias entre as espécies existentes [Izsák and Papp 2000].

$$
F=\sum_{i=0}^{N} \sum_{j=0}^{N} d_{i j}
$$


3. Distinção taxonômica média $(A v T D)$ : Representa a distância esperada entre dois indivíduos de diferentes espécies escolhidos ao acaso [Clarke and Warwick 1998].

$$
A v T D=\frac{\sum_{i=0}^{N} \sum_{j=0}^{N} d_{i j}}{\frac{S(S-1)}{2}}
$$

4. Distinção taxonômica total $(T T D)$ : Corresponde à distinção taxonômica média somada sobre todas as espécies [Clarke and Warwick 2001].

$$
T T D=\frac{\sum_{i=0}^{N} \sum_{j=0}^{N} d_{i j}}{(S-1)}
$$

5. Medida de pura diversidade $(D d)$ : Equivale à distância entre uma espécie $i$ e a espécie $j_{\min }$ mas próxima a ela, somada sobre todas as espécies [Weitzman 1992, Faith 1994].

$$
D d=\sum_{i=0}^{N} \sum_{j=0}^{N} d_{i j_{m i n}}
$$

\subsection{Seleção de atributos}

A etapa de seleção de atributos é muito utilizada em algoritmos de classificação para tentar aprimorar a eficácia do modelo final gerado. Com a presença de atributos redundantes ou pouco explicativos, o modelo pode perder a capacidade de generalização e ter sua acurácia comprometida. Assim, antes de cada etapa de treinamento foi aplicado o algoritmo de árvores extremamente aleatórias (extremely randomized trees) [Geurts et al. 2006] para calcular um peso representando a importância de cada atributo. Aqueles que apresentaram um peso maior do que a média foram mantidos e utilizados no treinamento.

\subsection{Classificação}

Foram testados três algoritmos distintos de aprendizagem de máquina para resolver o problema de classificação aqui proposto: floresta aleatória (random forest, RF) [Breiman 2001], máquina de vetores de suporte (support vector machine, SVM) [Cortes and Vapnik 1995] e redes neurais artificiais (artificial neural networks, ANN). A eficácia de cada um foi avaliada através de uma validação cruzada 5-fold, calculandose a média das métricas obtidas em cada fold. As métricas utilizadas foram a área sob a curva ROC (AUC, area under the curve), acurácia, sensibilidade e especificidade.

A etapa de seleção de atributos foi realizada em cada iteração da validação cruzada para selecionar aqueles a serem utilizados no treinamento. Como a seleção em cada fold pode dar origem a um conjunto distinto de atributos, na seção de resultados finais, são citados apenas os que foram escolhidas em 3 ou mais folds.

\section{Resultados}

A Tabela 2 apresenta as métricas referentes ao melhor resultado gerado por cada um dos algoritmos testados. Pode-se observar que o algoritmo SVM foi o que apresentou melhor desempenho na tarefa de classificação, com valores de AUC, acurácia, sensibilidade e especificidade de 0,926,0,902, 0,962 e 0,733, respectivamente. 
Tabela 2. Métricas do melhor resultado obtido por cada algoritmo testado, incluindo desvio-padrão.

\begin{tabular}{|c|c|c|c|c|}
\hline Algoritmo & AUC & Acurácia & Sensibilidade & Especificidade \\
\hline SVM & $0,926 \pm 0,025$ & $0,902 \pm 0,041$ & $0,962 \pm 0,028$ & $0,733 \pm 0,094$ \\
\hline RF & $0,907 \pm 0,063$ & $0,874 \pm 0,058$ & $0,948 \pm 0,038$ & $0,667 \pm 0,152$ \\
\hline ANN & $0,884 \pm 0,056$ & $0,891 \pm 0,030$ & $0,938 \pm 0,032$ & $0,760 \pm 0,090$ \\
\hline
\end{tabular}

Cada modalidade de MRI (T1, T1ce, T2 e FLAIR) deu origem a 68 atributos (17 índices calculados sobre quatro camadas distintas), totalizando 272 ao final da etapa de extração. O passo de seleção resultou na escolha de 47 atributos, levando em conta apenas aqueles escolhidos por 3 folds ou mais, conforme explicitado na seção 3.4. Mais de um terço dos atributos selecionados corresponde aos índices de McNaughton (I), Simpson $\left(D_{S}\right)$ e Shannon $\left(H^{\prime}\right)$. Além disso, entre os 47 atributos escolhidos, quase metade está relacionada à camada mais interna, o que sugere que a maior heterogeneidade de gliomas de alto grau é mais perceptível em regiões intratumorais mais próximas do núcleo da lesão.

Das quatro modalidades de MRI, T1ce foi a que esteve mais presente entre os atributos selecionados, possivelmente por ser um exame adquirido após a injeção endovenosa de contraste no paciente, que provoca uma hiperintensidade de sinal, permitindo a visualização de estruturas internas do tumor não vistas sem a presença de contraste [ETSUS 2012]. A Figura 5 exibe alguns exemplos de classificação produzidos pelo SVM, sendo todas as imagens mostradas correspondentes à modalidade T1ce, por possuir diferenças visualmente mais perceptíveis entre as classes. Observando as duas primeiras imagens correspondentes a gliomas de alto grau, pode-se notar que a imagem 5-b apresenta uma estrutura bem distinta da 5-a, com bordas mais suaves e uma textura mais homogênea, se assemelhando mais à imagem 5-c pertencente à classe LGG. Por outro lado, a imagem 5-d corresponde a um glioma de baixo grau, mas apresenta uma estrutura heterogênea com grande variação de escalas de cinza, estando mais próxima à estrutura apresentada por 5-a.

Tomando o índice de Simpson como exemplo, que mede a probabilidade de dois indivíduos escolhidos ao acaso pertencerem à mesma espécie - assumindo assim valores baixos em comunidades com maior diversidade de espécie (mais heterogêneas) e valores altos no caso contrário -, mesmo tendo se mostrado um índice com alto poder discriminativo, apresentou valores próximos entre 5-b e 5-c. Tal fato pode estar associado à presença de tons de cinza com menor variação e espalhados mais uniformemente pela imagem da lesão, o que acaba originando comunidades com espécies distribuídas de forma mais homogênea.

Através da mesma base de dados aqui aplicada, um modelo produzido por [Cho et al. 2018] utilizou o algoritmo RF com 5 atributos radiomics de forma e textura para resolver o mesmo problema de classificação, atingindo valores de AUC, acurácia, sensibilidade e especificidade de 0,9213, 0,8877, 0,9429 e 0,7333, respectivamente. Apesar de ter apresentado melhores resultados se comparado ao nosso modelo com RF, obteve um desempenho inferior aos resultados fornecidos por nosso modelo com SVM. A Ta- 
Tabela 3. Comparação de resultados para o problema de classificação de gliomas utilizando atributos radiomics em dados de MRI.

\begin{tabular}{|c|c|c|c|c|c|}
\hline Trabalho & AUC & Acurácia & Sensibilidade & Especificidade & $\begin{array}{c}\mathrm{N}^{o} \\
\text { de atributos }\end{array}$ \\
\hline [Zacharaki et al. 2009] & 0,896 & 0,878 & 0,846 & 0,955 & 24 \\
\hline [Zhang et al. 2017] & 0,960 & 0,960 & - & - & 50 \\
\hline [Cho and Park 2017] & 0,887 & 0,898 & 0,889 & 0,907 & $16-34$ \\
\hline [Chen et al. 2018] & 0,960 & 0,913 & 0,913 & - & 25 \\
\hline [Cho et al. 2018]* & 0,921 & 0,888 & 0,943 & 0,733 & 5 \\
\hline Método proposto & $\mathbf{0 , 9 2 6}$ & $\mathbf{0 , 9 0 2}$ & $\mathbf{0 , 9 6 2}$ & $\mathbf{0 , 7 3 3}$ & $\mathbf{4 7}$ \\
\hline
\end{tabular}

* Trabalhos com a mesma base de dados aqui utilizada.

bela 3 compara resultados obtidos em alguns trabalhos anteriores com os atingidos pela metodologia proposta.
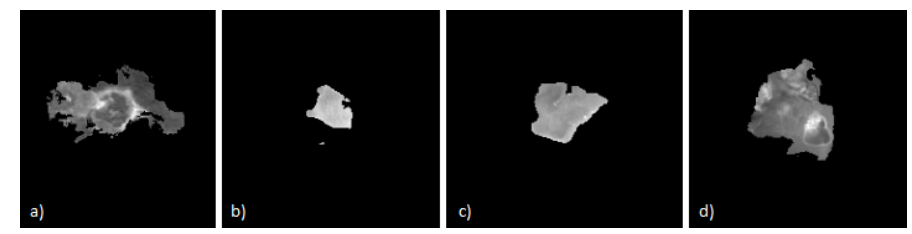

Figura 5. a) Tumor HGG classificado corretamente; d) Tumor HGG classificado como LGG; c) Tumor LGG classificado corretamente; d) Tumor LGG classificado como HGG.

\section{Conclusão}

A abordagem utilizada apresentou um bom desempenho em todos os algoritmos de classificação testados, mostrando que os atributos radiomics propostos possuem grande poder discriminativo no problema de classificação de gliomas entre HGG e LGG. O uso do radiomics por especialistas pode significar não apenas a otimização do tempo gasto na análise de exames de imagens, mas também o aumento da precisão com que tais análises são feitas.

Com a crescente disponibilidade de dados médicos digitalizados, já existem muitos estudos fornecendo soluções computacionais satisfatórias para diversos problemas existentes na medicina e o presente trabalho contribui para reforçar as vantagens associadas ao uso de uma abordagem radiomics neste contexto de imagens médicas, além de propor novos tipos de atributos para serem usados na classificação de gliomas.

\section{Referências}

Bakas, S., Akbari, H., Sotiras, A., Bilello, M., Rozycki, M., Kirby, J. S., Freymann, J. B., Farahani, K., and Davatzikos, C. (2017). Advancing The Cancer Genome Atlas glioma MRI collections with expert segmentation labels and radiomic features. Scientific data, 4(170117). doi:10.1038/sdata.2017.117.

Breiman, L. (2001). Random forests. Machine Learning, 45(1):5-32.

Campos, D. and Isaza, J. F. (2009). A geometrical index for measuring species diversity. Ecological Indicators, 9(4):651 - 658. ISSN: 1470-160X. doi: https://doi.org/10.1016/j.ecolind.2008.07.007. 
Chen, W., Liu, B., Peng, S., Sun, J., and Qiao, X. (2018). Computer-Aided Grading of Gliomas Combining Automatic Segmentation and Radiomics. International Journal of Biomedical Imaging, 2018(2512037):11.

Cho, H., Lee, S., Kim, J., and Park, H. (2018). Classification of the glioma grading using radiomics analysis. PeerJ 6:e5982. https://doi.org/10.7717/peerj.5982.

Cho, H. and Park, H. (2017). Classification of low-grade and high-grade glioma using multi-modal image radiomics features. volume 2017, pages 3081-3084. 39th Annual International Conference of the IEEE Engineering in Medicine and Biology Society (EMBC). 10.1109/EMBC.2017.8037508.

Clarke, K. and Warwick, R. (2001). Change in Marine Communities: An Approach to Statistical Analysis and Interpretation. Primer-E Ltd: Plymouth, UK.

Clarke, K. R. and Warwick, R. M. (1998). A taxonomic distinctness index and its statistical properties. Journal of Applied Ecology, 35(4):523-531. doi: 10.1046/j.13652664.1998.3540523.x.

Claus, E. B., Walsh, K. M., Wiencke, J. K., Molinaro, A. M., Wiemels, J. L., Schildkraut, J. M., Bondy, M. L., Berger, M., Jenkins, R., and Wrensch, M. (2015). Survival and low-grade glioma: the emergence of genetic information. Neurosurgical Focus FOC, $38(1)$.

Cortes, C. and Vapnik, V. (1995). Support-vector networks. Machine Learning, 20(3):273-297.

de Carvalho Filho, A. O., Silva, A. C., Cardoso de Paiva, A., Nunes, R. A., and Gattass, M. (2017). Computer-Aided Diagnosis of Lung Nodules in Computed Tomography by Using Phylogenetic Diversity, Genetic Algorithm, and SVM. Journal of Digital Imaging, 30(6):812-822. ISSN 1618-727X. https://doi.org/10.1007/s10278-017-99736.

ETSUS (2012). Meios de Contraste em RM. http://rle.dainf.ct.utfpr.edu.br/hipermidia/index.php/ressonancia-magnetica/meiosde-contraste-em-rm. Acesso em: dez. 2018.

Faith, D. (1994). Phylogenetic pattern and the quantification of organismal biodiversity. Philosophical transactions of the Royal Society of London. Series B, Biological sciences, 345:45-58.

Geurts, P., Ernst, D., and Wehenkel, L. (2006). Extremely randomized trees. Machine Learning, 63(1):3-42. ISSN 1573-0565. doi: 10.1007/s 10994-006-6226-1.

Gillies, R. J., Kinahan, P. E., and Hricak, H. (2016). Radiomics: Images Are More Than Pictures, They Are Data. Radiology, 278(2).

Gonzalez, R. C. and Woods, R. E. (2006). Digital Image Processing (3rd Edition). Prentice-Hall, Inc., Upper Saddle River, NJ, USA.

Izsák, J. and Papp, L. (2000). A link between ecological diversity indices and measures of biodiversity. Ecological Modelling, 130(1):151 - 156.

Karydis, M. and Tsirtsis, G. (1996). Ecological indices: A biometric approach for assessing eutrophication levels in the marine environment. Science of The Total Environment, 186:209-219. 10.1016/0048-9697(96)05114-5. 
Lamb, E., Bayne, E., Holloway, G., Schieck, J., Boutin, S., Herbers, J., and Haughland, D. (2009). Indices for monitoring biodiversity change: Are some more effective than others? Ecological Indicators, 9:432-444. doi: 10.1016/j.ecolind.2008.06.001.

Louis, D. N., Ohgaki, H., Wiestler, O. D., Cavenee, W. K., Burger, P. C., Jouvet, A., Scheithauer, B. W., and Kleihues, P. (2007). The 2007 who classification of tumours of the central nervous system. Acta Neuropathologica, 114(2):97-109.

Louis, D. N., Perry, A., Reifenberger, G., von Deimling, A., Figarella-Branger, D., Cavenee, W. K., Ohgaki, H., Wiestler, O. D., Kleihues, P., and Ellison, D. W. (2016). The 2016 world health organization classification of tumors of the central nervous system: a summary. Acta Neuropathologica, 131(6):803-820.

McGranahan, N. and Swanton, C. (2015). Biological and Therapeutic Impact of Intratumor Heterogeneity in Cancer Evolution. Cancer Cell, 28, Issue 1:141.

Menze, B. H. et al. (2015). The Multimodal Brain Tumor Image Segmentation Benchmark (BRATS). IEEE Transactions on Medical Imaging, 34(10):1993-2024. 10.1109/TMI.2014.2377694, 0278-0062.

Morris, E. K., Caruso, T., Buscot, F., Fischer, M., Hancock, C., Maier, T. S., Meiners, T., Müller, C., Obermaier, E., Prati, D., Socher, S. A., Sonnemann, I., Wäschke, N., Wubet, T., Wurst, S., and Rillig, M. C. (2014). Choosing and using diversity indices: insights for ecological applications from the german biodiversity exploratories. Ecology and Evolution, 4(18):3514-3524.

NBTS (2017). Brain Tumors By The Numbers. http://events.braintumor.org/wpcontent/uploads/2017/11/BrainTumorsBytheNumbers.pdf. Acesso em: dez. 2018.

Neto, A. C. d. S., Diniz, P. H. B., Diniz, J. O. B., Cavalcante, A. B., Silva, A. C., de Paiva, A. C., and de Almeida, J. D. S. (2018). Diagnosis of Non-Small Cell Lung Cancer Using Phylogenetic Diversity in Radiomics Context. In Campilho, A., Karray, F., and ter Haar Romeny, B., editors, Image Analysis and Recognition, pages 598-604, Cham. Springer International Publishing. 978-3-319-93000-8.

Weitzman, M. L. (1992). On diversity*. Quarterly Journal of Economics, 107(2):363405.

Zacharaki, E. I., Wang, S., Chawla, S., Yoo, D. S., Wolf, R., Melhem, E. R., and Davatzikos, C. (2009). Classification of Brain Tumor Type and Grade Using MRI. Magnetic Resonance in Medicine.

Zhang, X., Yan, L.-F., Yu-Chuan Hu, G. L., Yang, Y., Han, Y., Sun, Y., Liu, Z.-C., Tian, Q., Han, Z.-Y., Liu, L.-D., Hu, B.-Q., Qiu, Z.-Y., Wang, W., and Cui, G.-B. (2017). Optimizing a machine learning based glioma grading system using multi-parametric MRI histogram and texture features. Oncotarget. 